\title{
Adenylyl cyclase type 9 gene polymorphisms are associated with asthma and allergy in Brazilian children
}

\author{
Helena M.P. Teixeira ${ }^{a}$, Neuza M. Alcantara-Neves ${ }^{a}$, Maurício Barreto ${ }^{b}$, \\ Camila A. Figueiredo ${ }^{a}$, Ryan S. Costa ${ }^{a, *}$ \\ a Instituto de Ciências da Saúde, Universidade Federal da Bahia, Bahia, Brazil \\ b Instituto de Saúde Coletiva, Universidade Federal da Bahia, Bahia, Brazil
}

\section{A R T I C L E I N F O}

\section{Article history:}

Received 24 October 2016

Received in revised form

21 December 2016

Accepted 1 January 2017

Available online 8 January 2017

\section{Keywords:}

Allergy

Polymorphism

cAMP

$T$ regulatory cells

ADCY9

Asthma

\begin{abstract}
A B S T R A C T
Asthma is a chronic inflammatory disease of the respiratory tract. This heterogeneous disease is caused by the interaction of interindividual genetic variability and environmental factors. The gene adenylyl cyclase type 9 ( $A D C Y 9$ ) encodes a protein called adenylyl cyclase (AC), responsible for producing the second messenger cyclic AMP (cAMP). cAMP is produced by T regulatory cells and is involved in the down-regulation of T effector cells. Failures in cAMP production may be related to an imbalance in the regulatory immune response, leading to immune-mediated diseases, such as allergic disorders. The aim of this study was to investigate how polymorphisms in the $A D C Y 9$ are associated with asthma and allergic markers. The study comprised 1309 subjects from the SCAALA (Social Changes Asthma and Allergy in Latin America) program. Genotyping was accomplished using the Illumina 2.5 Human Omni bead chip. Logistic regression was used to assess the association between allergy markers and ADCY9 variation in PLINK 1.07 software with adjustments for sex, age, helminth infection and ancestry markers. The ADCY9 candidate gene was associated with different phenotypes, such as asthma, specific IgE, skin prick test, and cytokine production. Among 133 markers analyzed, 29 SNPs where associated with asthma and allergic markers in silico analysis revealed the functional impact of the 6 SNPs on ADCY9 expression. It can be concluded that polymorphisms in the $A D C Y 9$ gene are significantly associated with asthma and/or allergy markers. We believe that such polymorphisms may lead to increased expression of adenylyl cyclase with a consequent increase in immunoregulatory activity. Therefore, these SNPs may offer an impact on the occurrence of these conditions in admixture population from countries such as Brazil.
\end{abstract}

(C) 2017 Elsevier Ltd. All rights reserved.

\section{Introduction}

Asthma is a chronic disease of the airways that causes variable airflow obstruction in association with airway hyperresponsiveness and inflammation (Holgate, 2012). Asthma affects more than 334 million individuals worldwide (Global Asthma Network, 2014) and is considered a heterogeneous disease caused by the interaction of interindividual genetic variability and environmental factors (Miller and Ortega, 2013).

Usually, asthma symptoms begin in childhood in association with sensitization of the airways to common aeroallergens. Extracts of house dust mites, cockroaches, and animal and fungal allergens

\footnotetext{
* Corresponding author at: Instituto de Ciências da Saúde, Universidade Federal da Bahia, Avenida Reitor Miguel Calmon, s/n, Vale do Canela, CEP-40110-100, Salvador, Bahia, Brazil.

E-mail addresses: ryanscosta@yahoo.com.br, ryan.costa@ufba.br (R.S. Costa).
}

have proteolytic enzymes that can disrupt epithelial tight junctions and activate protease-activated receptors (Holgate, 2012). Atopic diseases are characterized by the presence of specific IgE to these aeroallergens. The Th2-type cytokines (interleukin (IL)-4, IL-5, IL9, and IL-13) orchestrate the allergic inflammatory cascade, which has a major role in allergic sensitization (Holgate, 2012; Deo et al., 2010).

The adenylyl cyclase type 9 (ADCY9) gene is located on chromosome 16 and codes for the protein adenyl cyclase (AC) type 9 , an integral membrane protein composed of twelve transmembrane segments. AC hydrolyses ATP in order to produce the second messenger cyclic AMP (adenosine-3', $5^{\prime}$-monophosphate), which subsequently activates the cAMP-dependent protein kinase A. It is responsible for the activation of multiple signal transduction pathways (Ortega, 2015; Sunahara et al., 2002).

Adenylyl cyclase type 9 is downstream of the $\beta 2$ adrenergic receptors $(\beta 2 A R)$, activating protein kinase $A$, which is responsible for phosphorylating multiple proteins in airway smooth muscle 


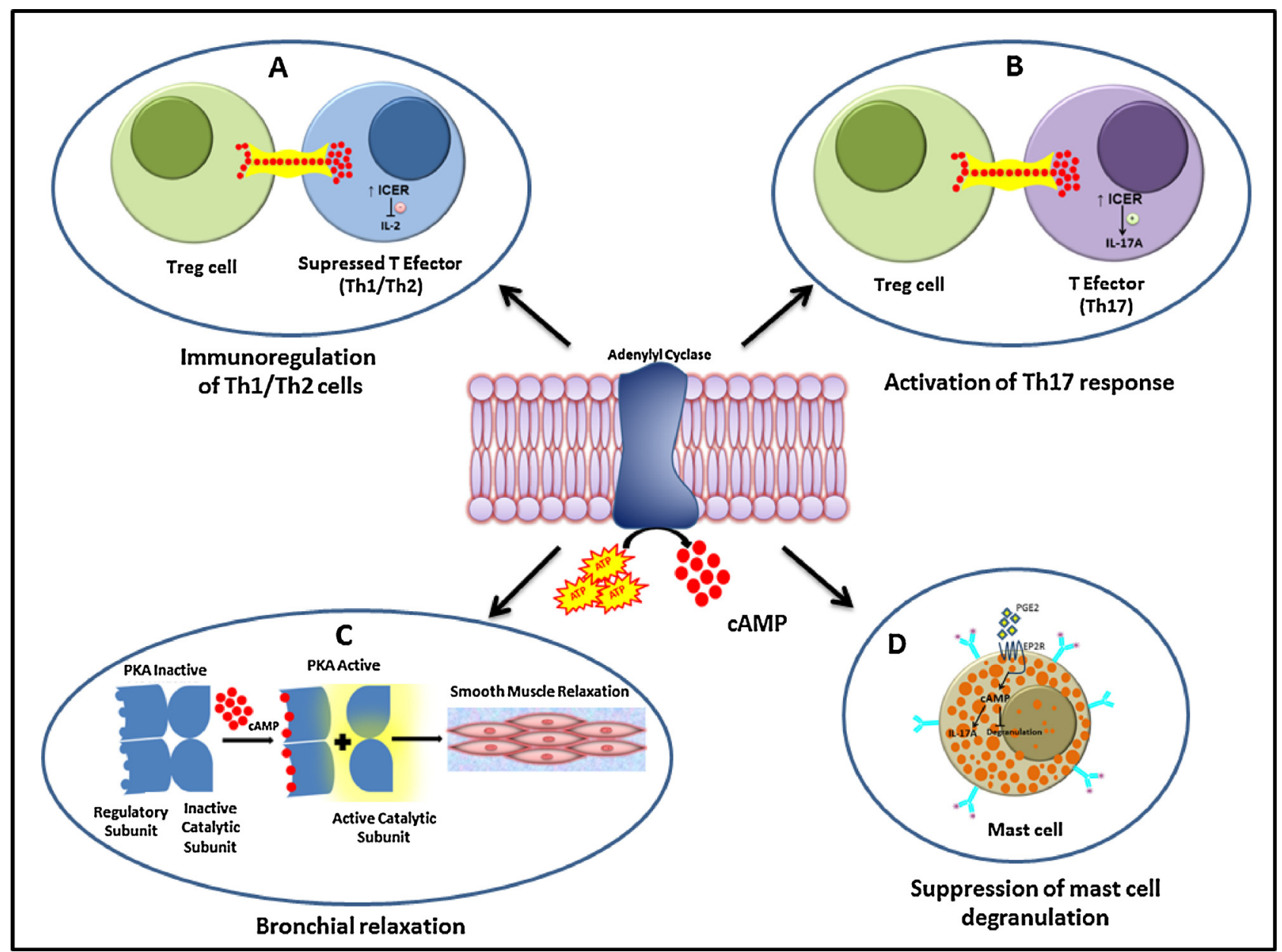

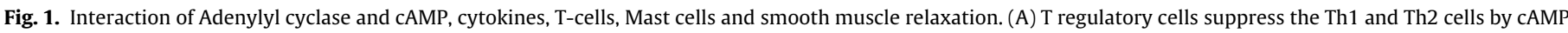

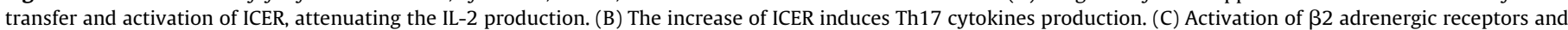

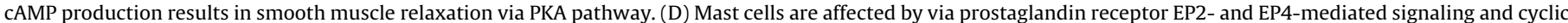
AMP pathways.

(Tantisira et al., 2005) (Fig. 1). This pathway represents an important target of $\beta$-agonist bronchodilator drugs, which are efficacious in improving asthma symptom control (Miller and Ortega, 2013).

Genetic polymorphisms in the ADCY9 gene have been demonstrated to be responsible for the therapeutic variability of $\beta 2$ adrenergic agonists in African-American and Caucasian children (Tantisira et al., 2005). The coding variant, Ile772Met (rs1042713), was associated with acute bronchodilation in response to a shortacting $\beta 2$ adrenergic agonist (SABA) in inhaled corticosteroids (ICS) treated asthmatics from a Korean asthmatic population (Miller and Ortega, 2013; Ortega, 2015). This polymorphism was previously identified by resequencing the $A D C Y 9$ coding regions where only one non-synonymous single nucleotide polymorphism was identified, which results in a substitution of isoleucine for the more common methionine at amino acid 772. The substitution lies in the $\mathrm{C} 1 \mathrm{~b}$ region of the catalytic domain of the enzyme (Tantisira et al., 2005) The Ile772Met substitution in the ADCY9 gene imparts enhanced $\beta 2 A R$ signal transduction in a corticosteroid-specific manner, and in asthma, the polymorphism is associated with enhanced $\beta$-agonist bronchodilator response under circumstances when corticosteroids are co-administered (Miller and Ortega, 2013; Tantisira et al., 2005).

In addition to $\beta 2 \mathrm{ARs}$, considering asthma and other allergies as immune-mediated diseases, disturbances in regulatory mechanisms could also play a role in the occurrence of such diseases. Thus, the activity of $\mathrm{T}$ regulatory (Treg) cells may be of impor- tance. Treg cells harbor, in fact, high levels of intracellular cAMP, which are increased even further after T-cell receptor (TCR) stimulation and/or CD4 engagement (Bodor et al., 2012). The suppressive potency of Treg cells has been shown to depend on cAMP, which induces ICER (inducible cyclic cAMP early repressor), This repressor preferentially inhibits the production of IL-2, an essential growth factor for T-effector cells (Bodor et al., 2012). On the other hand, the forced expression of ICER in human T cells enhances cytokines expression the Th17 cells such as IL-17A (Yoshida et al., 2016) (Fig. 1).

The expression of cAMP-degrading phosphodiesterase 3b (PDE3b) is reduced in Treg cells by direct binding of Forkhead box P3 (Foxp3) to the PDE3b gene (Huang et al., 2009). Thus, Foxp3, the master regulator of Treg cells, is responsible for elevated levels of cAMP and downregulates miR-142-3p, which silences ADCY9 mRNA, also leading to upregulated cAMP production in Treg cells. The microRNA miR-142-3p regulates $A D C Y 9$ expression at the translational level and the production of cAMP in these two T-cell subsets (Bodor et al., 2012; Huang et al., 2009).

Though the cAMP level is related to immunoregulatory activity, there are controversies about the cAMP-dependent immunosuppression of the Th2 subset. Mosenden and Taskén (2011) showed that similar amounts of cAMP have a stronger suppressive effect on the Th1 response mediated by interferon- $\gamma$ (IFN $\gamma$ ), IL-12, and IL2 as well as tumor necrosis factor- $\alpha$ (TNF $\alpha$ ) production, whereas Th2 cell effector functions remained unaffected and the levels of 
cytokines IL-4, IL-5, IL-6, and IL-13 in Th2 cells remain unchanged when compared with those in Th1 cells (Mosenden and Taskén, 2011). This is attributed to the ability of Th2 cells to produce cytokines, which sustain their own proliferation by other mechanisms, even in the absence of responsiveness to IL-2 (Dehzad et al., 2016).

On the other hand, Verjans et al. (2015) shows that IL-2 is involved in Th2 cell priming as well as in the differentiation and maintenance of the differentiated state. Both the cAMP response element binding protein (CREB) and cAMP-responsive element modulator (CREM) are activated by the second messenger cAMP, which is directly involved in the regulation of Th2-type cytokines, such as IL-5 (Verjans et al., 2015).

Studies conducted thus far on $A D C Y 9$ polymorphisms in asthma have explored the association between lack of response to bronchodilators and treatment in asthmatic patients. Therefore, the aim of this study was to investigate the association of polymorphisms in the ADCY9 gene with asthma and markers of allergy.

\section{Methods}

\subsection{Study population and data collection}

The study population included 1309 unrelated children between 4 and 11 years old from the city of Salvador, Northeastern Brazil, which has a population of 2.9 million. Over $80 \%$ of the population is of African descent or mixed-race (mulatto). The methods of this study have been reported elsewhere (Alcantara-Neves et al., 2011). The children were surveyed in 2005 to collect data on asthma status; blood samples were obtained to evaluate infection, total and specific IgE, and immunological makers; and stool samples were tested for helminth and protozoan infections. Data were collected, including information on wheezing and risk factors for wheezing and allergic diseases, using a Portuguese-adapted ISAAC (International Study of Asthma and Allergies in Childhood) Phase II questionnaire (Alcantara-Neves et al., 2011). All population data used in this study are from the SCAALA database.

Table 1 summarizes the characteristics of the study population. We observed equal proportions of asthmatic subjects across all age groups. Differences in markers of allergy, such as SPT reactivity, specific IgE levels, and atopic asthma, were statistically significant $(P<0.0001)$ between non asthmatic and asthmatic groups.

Ethical approval was obtained from the Brazilian National Ethical Committee in 2005 and the Ethical Committee of the Collective Health Institute, Bahia Federal University (in annex). Written informed consent was obtained from the legal guardian or parents of each subject. It details all of the procedures in the course of the project.

\subsection{Asthma definition}

Children were classified as having current wheeze using a Portuguese-adapted ISAAC and were considered to have current asthma symptoms if parents reported at least one of the following: (1) asthma diagnosis by a doctor; (2) wheezing with exercise in the last 12 months; (3) 4 or more episodes of wheezing in the last 12 months; and (4) waking up at night because of wheezing in the last 12 months. Atopic and nonatopic asthma was defined as symptoms of asthma in the presence or absence, respectively, of a serum IgE level of $0.70 \mathrm{kU} / \mathrm{L}$ or greater for any of the tested aeroallergens (Alcantara-Neves et al., 2011).

\subsection{Allergic markers}

The following tests were previously performed by AlcantaraNeves et al. (2011) and Barreto et al. (2006). Skin prick tests
(SPTs) were performed on the right forearms of the children using standardized extracts (ALK-Abelló, São Paulo, Brazil) for six common aeroallergens: Blomia tropicalis, Dermatophagoides pteronyssinus, Blattella germanica, Periplaneta americana, and cat and dog epithelium. Saline and $10 \mathrm{mg} / \mathrm{mL}$ histamine solution were used as negative and positive controls, respectively. The reaction was read after $15 \mathrm{~min}$. A reaction was considered positive if the wheal size was at least $3 \mathrm{~mm}$ greater than that elicited by the negative control (Barreto et al., 2006).

Blood was collected and sera were kept frozen until use. Specific IgE (sIgE) anti-mite (D. pteronyssinus and B. tropicalis) and anti-cockroaches ( $P$. americana and $B$. germanica) in serum was measured with the Pharmacia ImmunoCAP System IgE FEIA (Pharmacia, Uppsala, Sweden), according to the manufacturer's instructions. Results of sIgE measurement were considered positive in a child if levels of $0.70 \mathrm{kU} / \mathrm{L}$ or greater were detected for at least one of the four tested allergens (Alcantara-Neves et al., 2011).

Whole blood was cultivated in a $5 \% \mathrm{CO}_{2}$ atmosphere for $24 \mathrm{~h}$ for the detection of IL-10 and for 5 days for the detection of IL-5, IL-13, and IFN- $\gamma$. Cytokines in supernatant fluids were detected using Pharmigen BD antibody pairs and recombinant standards (Pharmigen, San Diego, Ca, USA) by capture ELISA following the manufacturer's instructions (Barreto et al., 2006).

\subsection{DNA extraction and genotyping}

DNA was extracted from peripheral blood using a QIAGEN kit (Gentra Puregene Blood Kit; Hilden, Germany) following the manufacturer's recommendations. Samples from 1309 subjects of the SCAALA (Social Changes Asthma and Allergy in Latin America) program were genotyped by Illumina using the Illumina 2.5 Human Omni bead chip, which is a wide scanning platform with 2.5 million markers genotyped throughout the entire genome. For this study, genetic information was extracted from positions 4012650-4166186 located on chromosome 16 (16p13.3 cytogenetic location).

\subsection{Statistical analysis}

Quality control for all SNPs was carried out in stages using PLINK version 1.07 using the following parameters: deviation from HardyWeinberg equilibrium (HWE) with a P-value of less than $10^{-3}$, minor allele frequency (MAF) of less than $5 \%$, and percentage of missing loci more than 1\%. Of the 194 SNPs genotyped, 133 passed the quality control and were included in the analysis. Association analyses were conducted using PLINK 1.07 (Purcell et al., 2007). The analyses were adjusted for sex, age, helminth infection, and principal components (PC) 1 and 2. Principal component analysis was carried out and its first two components (PC1 to African and PC2 to European) were used as covariates to control confounding by population structure (Costa et al., 2015). The helminth infection data were obtained from a prior study by Alcantara-Neves et al. (2011).

Multivariate logistic regression analyses for SNPs that passed quality control were carried out under an additive model. The asymptotic $P$-value was observed, and the odds ratio (OR) was estimated. Additionally, the permutation procedures (Perm) were calculated to provide a computationally intensive approach to generating significance levels. To solve the problem of multiple comparisons this has been managed with methods that control the false discovery rate such as permutation test (Lage-Castellanos et al., 2010), this test preserve the correlational structure between SNPs, (Purcell et al., 2007).

Linkage disequilibrium (LD) refers to the association of alleles on the same chromosome but at different loci. These associations could be affected by mutation, recombination, gene conversion, selection, genetic drift, or demographic factors, such as inbreeding, migration, 
Table 1

Characteristics of the SCAALA population according to asthma status and the variables included in the study.

\begin{tabular}{|c|c|c|c|c|c|}
\hline & \multicolumn{2}{|c|}{ Non asthmatic $(\mathrm{n}=950)$} & \multicolumn{2}{|c|}{ Asthmatic $(\mathrm{n}=277)$} & P value \\
\hline \multicolumn{6}{|l|}{ Age } \\
\hline$\leq 5 \mathrm{y}$ & 325 & $34.21 \%$ & 96 & $34.66 \%$ & \\
\hline $6-7 y$ & 313 & $32.95 \%$ & 99 & $35.74 \%$ & \\
\hline$\geq 8 \mathrm{y}$ & 292 & $30.74 \%$ & 78 & $28.16 \%$ & 0.083 \\
\hline \multicolumn{6}{|l|}{ Sex } \\
\hline Male & 516 & $54.32 \%$ & 143 & $51.62 \%$ & \\
\hline Female & 414 & $43.58 \%$ & 130 & $46.93 \%$ & 0.029 \\
\hline Atopic asthma & 0 & $0.00 \%$ & 134 & $48.38 \%$ & $<0.0001$ \\
\hline IgE specific for at least one allergen & 327 & $34.42 \%$ & 134 & $48.38 \%$ & $<0.0001$ \\
\hline sIgE for Blatella germanica & 118 & $12.42 \%$ & 47 & $16.97 \%$ & $<0.0001$ \\
\hline sIgE for Blomia tropicalis & 289 & $30.42 \%$ & 129 & $46.57 \%$ & $<0.0001$ \\
\hline sIgE for Dermatophagoides pteronyssinus & 179 & $18.84 \%$ & 91 & $32.85 \%$ & $<0.0001$ \\
\hline sIgE for Periplaneta americana & 81 & $8.53 \%$ & 31 & $11.19 \%$ & $<0.0001$ \\
\hline Skin test for at least one tested allergen & 273 & $28.74 \%$ & 99 & $35.74 \%$ & $<0.0001$ \\
\hline Skin test for Dermatophagoides pteronyssinus & 132 & $13.89 \%$ & 62 & $22.38 \%$ & $<0.0001$ \\
\hline Skin test for Blomia tropicalis & 193 & $20.32 \%$ & 76 & $27.44 \%$ & $<0.0001$ \\
\hline Skin test for Periplaneta americana & 120 & $12.63 \%$ & 49 & $17.69 \%$ & $<0.0001$ \\
\hline Skin test for Blatella germanica & 71 & $7.47 \%$ & 31 & $11.19 \%$ & $<0.0001$ \\
\hline Skin test for dog epithelium & 7 & $0.74 \%$ & 6 & $2.17 \%$ & $<0.0001$ \\
\hline Skin test for cat epithelium & 6 & $0.63 \%$ & 3 & $1.08 \%$ & $<0.0001$ \\
\hline IL-5 unstimulated production & 45 & $4.74 \%$ & 15 & $5.42 \%$ & $<0.0001$ \\
\hline IL-13 Unstimulated production & 313 & $32.95 \%$ & 84 & $30.32 \%$ & $<0.0001$ \\
\hline
\end{tabular}

Table 2

Base pairs, alleles, minor allele frequency, Hardy-Weinberg equilibrium, function and RegulomeDB score of ADCY9 SNPs.

\begin{tabular}{|c|c|c|c|c|c|}
\hline SNP & Location allele $^{a}$ & MAF & HWE & Function $^{\mathrm{b}}$ & RegulomeDB Score \\
\hline kgp12201986 & $\mathrm{A} / \mathrm{G}$ & 0.32 & 0.80 & - & - \\
\hline rs1045476 & $\mathrm{A} / \mathrm{G}$ & 0.46 & 0.44 & intron variant, utr variant 3 prime & - \\
\hline rs13337675 & $\mathrm{G} / \mathrm{A}$ & 0.19 & 0.11 & intron variant & 5 \\
\hline rs2058166 & $\mathrm{A} / \mathrm{G}$ & 0.18 & 0.35 & Intron variant & 5 \\
\hline rs2108987 & $\mathrm{G} / \mathrm{A}$ & 0.21 & 0.87 & Intron variant & - \\
\hline rs2239313 & $\mathrm{C} / \mathrm{G}$ & 0.42 & 0.11 & intron variant & 5 \\
\hline rs2283497 & $\mathrm{T} / \mathrm{G}$ & 0.44 & 0.78 & intron variant & 4 \\
\hline rs2444217 & $\mathrm{T} / \mathrm{C}$ & 0.33 & 1.00 & intron variant & $2 \mathrm{a}$ \\
\hline rs2531995 & $\mathrm{A} / \mathrm{G}$ & 0.34 & 0.62 & intron variant, utr variant 3 prime & 1f \\
\hline rs2532019 & $\mathrm{C} / \mathrm{A}$ & 0.48 & 0.29 & intron variant & 5 \\
\hline rs2601777 & $\mathrm{A} / \mathrm{G}$ & 0.45 & 0.09 & intron variant & 5 \\
\hline rs2601796 & $\mathrm{C} / \mathrm{T}$ & 0.46 & 0.98 & intron variant & - \\
\hline rs2601798 & $\mathrm{C} / \mathrm{G}$ & 0.09 & 0.62 & Intron variant & 5 \\
\hline rs2601807 & $\mathrm{T} / \mathrm{C}$ & 0.14 & 0.17 & Intron variant & 5 \\
\hline rs2601814 & $\mathrm{G} / \mathrm{T}$ & 0.49 & 0.59 & intron variant & 6 \\
\hline rs2601831 & $\mathrm{A} / \mathrm{G}$ & 0.49 & 0.82 & Intron variant & 5 \\
\hline rs409963 & $\mathrm{G} / \mathrm{A}$ & 0.11 & 0.13 & Intron variant & 4 \\
\hline rs432166 & $\mathrm{T} / \mathrm{G}$ & 0.36 & 0.34 & Intron variant & 5 \\
\hline rs4785947 & $\mathrm{A} / \mathrm{G}$ & 0.13 & 0.80 & Intron variant & 6 \\
\hline rs4785951 & $\mathrm{C} / \mathrm{T}$ & 0.35 & 0.63 & Intron variant & 6 \\
\hline rs4785953 & $\mathrm{G} / \mathrm{A}$ & 0.23 & 0.06 & intron variant & - \\
\hline rs61042195 & $\mathrm{G} / \mathrm{C}$ & 0.21 & 0.08 & Intron variant & 6 \\
\hline rs6500567 & $\mathrm{G} / \mathrm{A}$ & 0.26 & 0.83 & Intron variant & 4 \\
\hline rs7196832 & $\mathrm{G} / \mathrm{A}$ & 0.50 & 0.38 & Intron variant & 5 \\
\hline rs73490519 & $\mathrm{A} / \mathrm{G}$ & 0.07 & 1 & Intron variant & - \\
\hline rs79964474 & $\mathrm{T} / \mathrm{C}$ & 0.07 & 0.68 & Intron variant & 5 \\
\hline rs8045426 & $\mathrm{T} / \mathrm{C}$ & 0.08 & 0.37 & Intron variant & 5 \\
\hline rs8061182 & $\mathrm{C} / \mathrm{T}$ & 0.19 & 0.59 & intron variant & 6 \\
\hline rs9922384 & $\mathrm{G} / \mathrm{A}$ & 0.17 & 1.00 & intron variant & $2 b$ \\
\hline
\end{tabular}

a First is the alternative allele and the second is the reference allele $(1 / 2)$

b NCBI (National Center for Biotechnology Information).

and population structure (Wang et al., 2006). The Haploview 4.2 software was used to calculate the degree of confidence in the D' value (Barrett et al., 2005).

\subsection{In silico analysis}

Information from the National Center for Biotechnology Information (NCBI) was obtained on the function of each SNP (www.ncbi.nlm.nih.gov).

RegulomeDB is a database for the interpretation of regulatory variants in the human genome. It includes high-throughput, experimental data sets from ENCODE (Encyclopedia of DNA Elements) and other sources. RegulomeDB (regulomedb.org) identifies putative regulatory potential and functional variants through computational predictions and manual annotations (Boyle et al., 2012). This database has a score that ranges from 1 to 6 (Supplementary material-Table S8). Increasing of evidence for a variant to be located in a functional region is indicated by lower scores. Scores of 1 a to $1 \mathrm{f}$ indicate that the variant is likely to affect binding and linked to the expression of a gene target, whereas scores of $2 a$ to $2 c$ indicate only that the variant is likely to affect binding. Variants with scores of $3 a$ and $b$ are less likely to affect binding, and scores of 4, 5, and 6 
Table 3

Significant associations between $A D C Y 9$ SNPs and asthma by logistic regression adjusted for sex, age, helminth infections, and principal components 1 and 2 .

\begin{tabular}{lllll}
\hline SNP & OR & $95 \% \mathrm{CI}$ & $P$-value & Perm \\
\hline rs2601796 & 1.31 & $1.07-1.60$ & 0.009 & 0.010 \\
rs2601814 & 1.27 & $1.04-1.55$ & 0.019 & 0.016 \\
\hline
\end{tabular}

indicate minimal binding evidence or no available data (Boyle et al., 2012).

The Genotype-Tissue Expression Project GTEx (www.gtexportal.org) of the National Institutes of Health Common Fund has established a resource database and associated tissue bank with the objective of studying the relationship between gene expression and genetic variation as well as other molecular phenotypes in multiple reference human tissues (The GTEx Consortium, 2014). Expression quantitative trait loci (eQTL) mapping offers a powerful approach to elucidate the genetic component underlying altered gene expression. Genetic variation can also influence gene expression through alterations in splicing, noncoding RNAs, and RNA stability. Gene expression is differentially regulated across tissues, and many human transcripts are expressed in a limited set of cell types or during a limited developmental stage (The GTEx Consortium, 2014). From this project information was extracted about the functional impact of $A D C Y 9$ polymorphisms.

\section{Results}

\subsection{Description of the ADCY9 polymorphisms}

In this study, 194 SNPs were extracted from the Illumina genotyping chip, in 1309 subjects. Of these markers, one was excluded by the test for Hardy-Weinberg Equilibrium (HWE) $\left(p \leq 10^{-3}\right)$ and 60 SNPs were excluded due to very low MAF $(<0.05)$. No sample was excluded by low genotyping (mind $>0.1$ ) or missingness (geno >0.1). Thus, of 194 markers, 133 SNPs were included in the association analysis. Table 2 shows information from 29 SNPs discussed in the present study and presents SNPs with at least two associations with allergy or asthma phenotypes; the other 104 are shown in the Supplementary material (Table A.1) with only one association (Table A.2).

\subsection{Linkage disequilibrium}

Linkage disequilibrium (LD) analysis was carried out using the Haploview program. There are nine blocks in LD (Fig. 2).

\subsection{Association of ADCY9 SNPs with asthma}

The asthma phenotypes were identified by the asthma definition presented in the methodology item. The SNPs rs2601796 (OR $1.31,1.07-1.60$ ) and rs2601814 (OR 1.27, 1.04-1.55) were positively associated with asthma in the additive model (Table 3 ).

\subsection{Association of ADCY9 SNPs with atopic asthma}

Three SNPs were positively associated with allergy in asthmatic patients and only rs2283497 (OR 0.67, 0.46-0.96) was negatively associated with allergy in asthmatic patients (Table 4).

\subsection{Association of ADCY9 SNPs with sIgE levels}

The results of the analysis between the ADCY9 SNPs and serumspecific IgE are shown in Table 5. The SNP kgp12201986 (OR 1.25, 1.04-1.49) was positively associated and the SNPs rs432166 (OR $0.83,0.70-0.99$ ) and rs2601777 (OR 0.80, 0.67-0.95) were nega-
Table 4

Significant association between $A D C Y 9$ SNPs and allergy in asthmatic subjects by logistic regression adjusted for sex, age, helminth infections, and principal components 1 and 2 .

\begin{tabular}{lllll}
\hline SNP & OR & $95 \%$ CI & P Value & Perm \\
\hline rs8061182 & 1.84 & $1.12-3.03$ & 0.017 & 0.012 \\
rs4785947 & 1.84 & $1.08-3.13$ & 0.025 & 0.031 \\
kgp12201986 & 1.54 & $1.03-2.31$ & 0.035 & 0.044 \\
rs2283497 & 0.67 & $0.46-0.96$ & 0.031 & 0.041 \\
\hline
\end{tabular}

Table 5

Significant association between $A D C Y 9$ SNPs and sIgE by logistic regression adjusted for sex, age, helminth infections, and principal components 1 and 2 .

\begin{tabular}{|c|c|c|c|c|}
\hline SNP & OR & $95 \% \mathrm{CI}$ & P value & Perm \\
\hline \multicolumn{5}{|c|}{ IgE specific for at least one allergen } \\
\hline kgp12201986 & 1.25 & $1.04-1.49$ & 0.015 & 0.012 \\
\hline rs432166 & 0.83 & $0.70-0.99$ & 0.040 & 0.040 \\
\hline rs2601777 & 0.80 & $0.67-0.95$ & 0.010 & 0.010 \\
\hline \multicolumn{5}{|c|}{ sIgE for Blomia tropicalis } \\
\hline kgp12201986 & 1.31 & $1.09-1.57$ & 0.004 & 0.004 \\
\hline rs2 2444217 & 1.26 & $1.05-1.51$ & 0.012 & 0.016 \\
\hline rs4785947 & 1.31 & $1.02-1.67$ & 0.036 & 0.034 \\
\hline rs2601777 & 0.81 & $0.68-0.97$ & 0.019 & 0.027 \\
\hline rs2283497 & 0.83 & $0.70-0.98$ & 0.031 & 0.037 \\
\hline rs4785953 & 0.81 & $0.66-0.99$ & 0.036 & 0.037 \\
\hline rs8045426 & 0.72 & $0.52-0.98$ & 0.038 & 0.038 \\
\hline rs432166 & 0.83 & $0.69-0.98$ & 0.036 & 0.042 \\
\hline \multicolumn{5}{|c|}{ sIgE for Dermatophagoides pteronyssinus } \\
\hline rs2283497 & 0.81 & $0.66-0.98$ & 0.032 & 0.025 \\
\hline rs6500567 & 0.76 & $0.61-0.96$ & 0.020 & 0.027 \\
\hline rs4785953 & 0.79 & $0.63-1.00$ & 0.048 & 0.040 \\
\hline
\end{tabular}

tively associated with IgE for at least one allergen. Three SNPs were positively associated and five SNPs were negatively associated with $B$. tropicalis SIgE. Three SNPs were negatively associated with $D$. pteronyssinus SIgE.

\subsection{Association of ADCY9 SNPs with skin prick test}

The results of the analysis between ADCY9 SNPs and the skin prick test are shown in Table 6. Nine SNPs were positively associated and six were negatively associated with the skin prick test for at least one tested allergen.

Analysis of polymorphisms and the specific skin test for $D$. pteronyssinus revealed that nine SNPs were positively associated and ten SNPs were negatively associated with this test. Six SNPs were positively associated with the skin prick test for Blomia tropicalis.

Six SNPs were positively associated and five were negatively associated with the skin prick test for $P$. americana. Two SNPs were positively associated and rs13337675 (OR 0.58, 0.22-0.37) was negatively associated with the skin prick test for B. germanica.

Two SNPs were positively associated and rs2108987 (OR 0.12, $0.02-0.93$ ) was negatively associated with the skin prick test for dog epithelium. The rs7196832 (OR 3.02, 1.10-8.29) was positively associated with the skin prick test for cat epithelium (Table 6).

\subsection{Association of ADCY9 SNPs with cytokine level production}

Four SNPs were positively associated with unstimulated production of IL-5, and rs13337675 (OR 0.46, 0.25-0.86) was negatively associated with unstimulated production of IL-5 (Table 7).

Two SNPs were positively associated with unstimulated production of IL-13 (rs2531995 and rs8045426), and rs1045476 (OR $0.83,0.70-0.99$ ) was negatively associated with unstimulated production of IL-13 (Table 7). The cytokines IFN- $\gamma$ and IL-10 were 


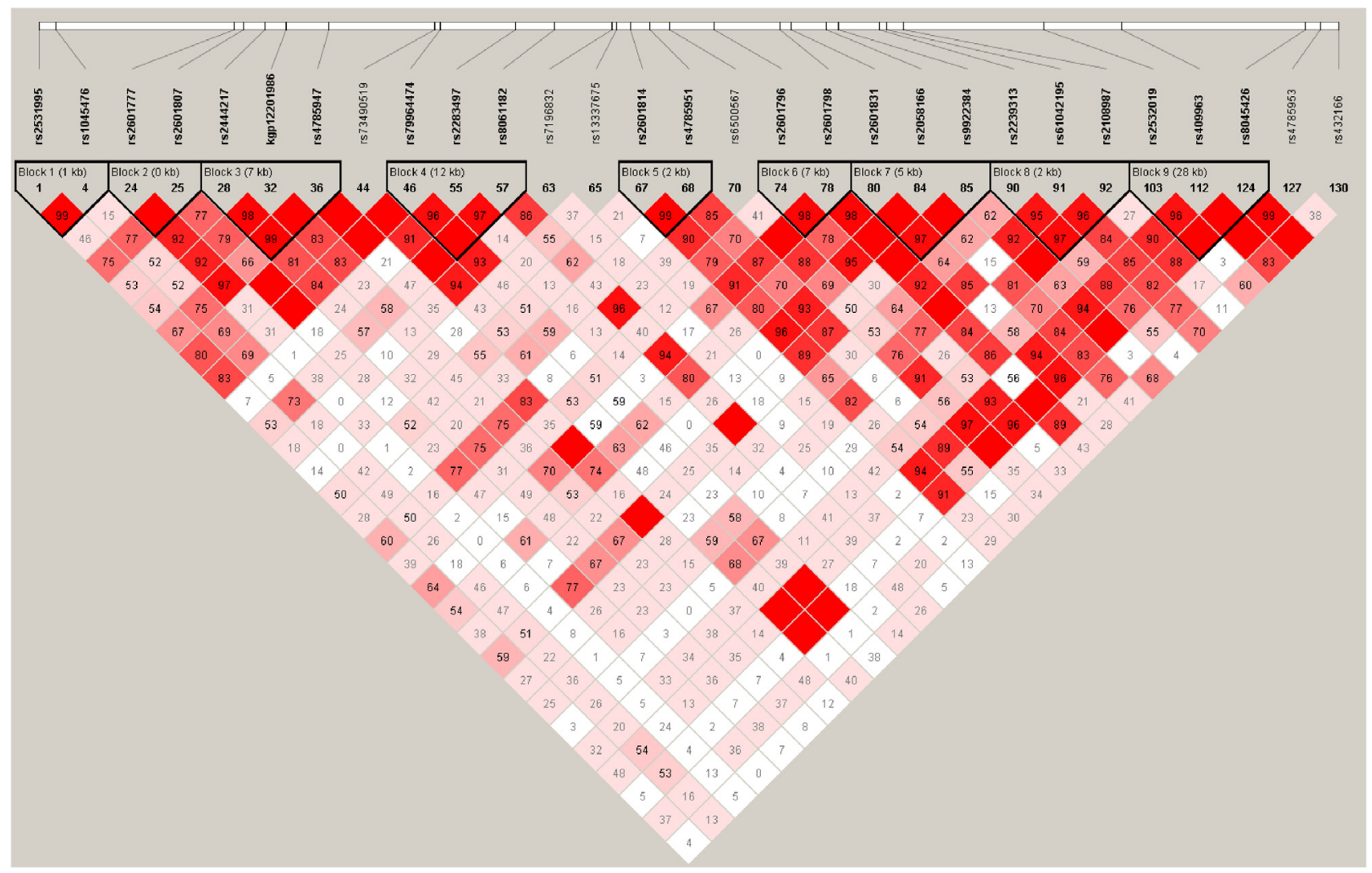

Fig. 2. Pairwise LD within Haploview using the $\mathrm{D}^{\prime}$ squared statistic for the $A D C Y 9$ gene. Intensity of shading indicates the degree of confidence in the $\mathrm{D}^{\prime}$ value.

not statistically significantly associated with any polymorphisms of $A D C Y 9$ in this study.

\subsection{GTEx in silico analyses}

Using the GTEx browser we found the rs2108987 $(\mathrm{p}<0.001)$, rs2239313 ( $\mathrm{p}<0.001)$ rs2531995 ( $\mathrm{p}<0.001), \mathrm{rs} 409963(\mathrm{p}<0.001)$, rs2532019 ( $<<0.001)$, and rs8045426 $(\mathrm{p}<0.001)$ associated with differential expression of $A D C Y 9$ in whole blood or thyroid tissue (The GTEx Consortium, 2014) (Fig. 3).

\section{Discussion}

This is the first study to examine the genetic association between variants of the ADCY9 gene with asthma and allergy. Our data indicate that the ADCY9 polymorphisms rs2601796 and rs2601814 were associated with an increase in the risk of asthma, whereas other SNPs are associated with allergy in children.

The ADCY9 expression is directly related to cAMP production (Fig. 1), and high intracellular cAMP levels in regulatory $\mathrm{T}$ cells are critically involved in contact-dependent suppression of conventional Th1 and Th2 cells and the enhance of IL-17 production (Yoshida et al., 2016; Bodor et al., 2012) (Fig. 1).

The SNPs rs2601796 and rs2601814 were negatively associated with several markers of allergy. In addition, both SNPs were positively associated with asthma. Although there is no description of the impact of these SNPs on gene expression, we hypothesize that these variants may lead to increased expression of $A D C Y 9$ with consequent modulation of allergy (Bodor et al., 2012) in parallel to an increase of Th17 response (Yoshida et al., 2016), which may explains the positive association with asthma and, in this case, probably, non-allergic asthma. However, further studies are needed to better explore the role of these two controversial markers on asthma and allergy.

In this study, the $C$ allele of rs2239313 was positively associated with skin test for at least one of the following allergens: $D$. pteronyssinus, B. tropicalis, P. americana, and dog epithelium as well as sIgE for $D$. pteronyssinus. Data from the GTEx project show that the reference genotype $\mathrm{G} / \mathrm{G}$ is associated with higher $A D C Y 9$ expression than the alternative genotype $\mathrm{C} / \mathrm{C}$, which is associated with low expression in whole blood (Fig. 3B).

The A allele of rs2531995 was positively associated with skin test for at least one of the following allergens: $D$. pteronyssinus, $B$. tropicalis, and $P$. americana as well as unstimulated production of IL-13. GTEx data, allele A expressed less $A D C Y 9$ than the reference allele G (Fig. 3C) in thyroid tissue. Furthermore, this SNP rs2531995 had a score of $1 \mathrm{f}$ from RegulomeDB, meaning that this polymorphism is likely to affect binding and linked to the expression of a gene target.

We also found the $G$ allele of rs409963 positively associated with skin test for at least one allergen and $P$. americana. This allele was associated with lower $A D C Y 9$ expression than the reference $A$ allele (Fig. 3D).

The $C$ allele of rs2532019 was negatively associated with skin test for $D$. pteronyssinus and for $P$. americana. Analyzing the expression level in the GTEx, the $C$ allele increases $A D C Y 9$ expression in whole blood (Fig. 3E). Thus, individuals with the $\mathrm{C} / \mathrm{C}$ genotype had higher cAMP production.

These data suggest that genetic variants in ADCY9 were highly involved in allergic status. A report by Bodor et al. (2012) reinforces the possibility that reduced adenylyl expression and the consequent decrease in cAMP production, which is well established as a potent negative regulator of T-cell immune function (Mosenden and Taskén, 2011), had been negatively associated with a regulatory activity from Treg cells that may contribute 

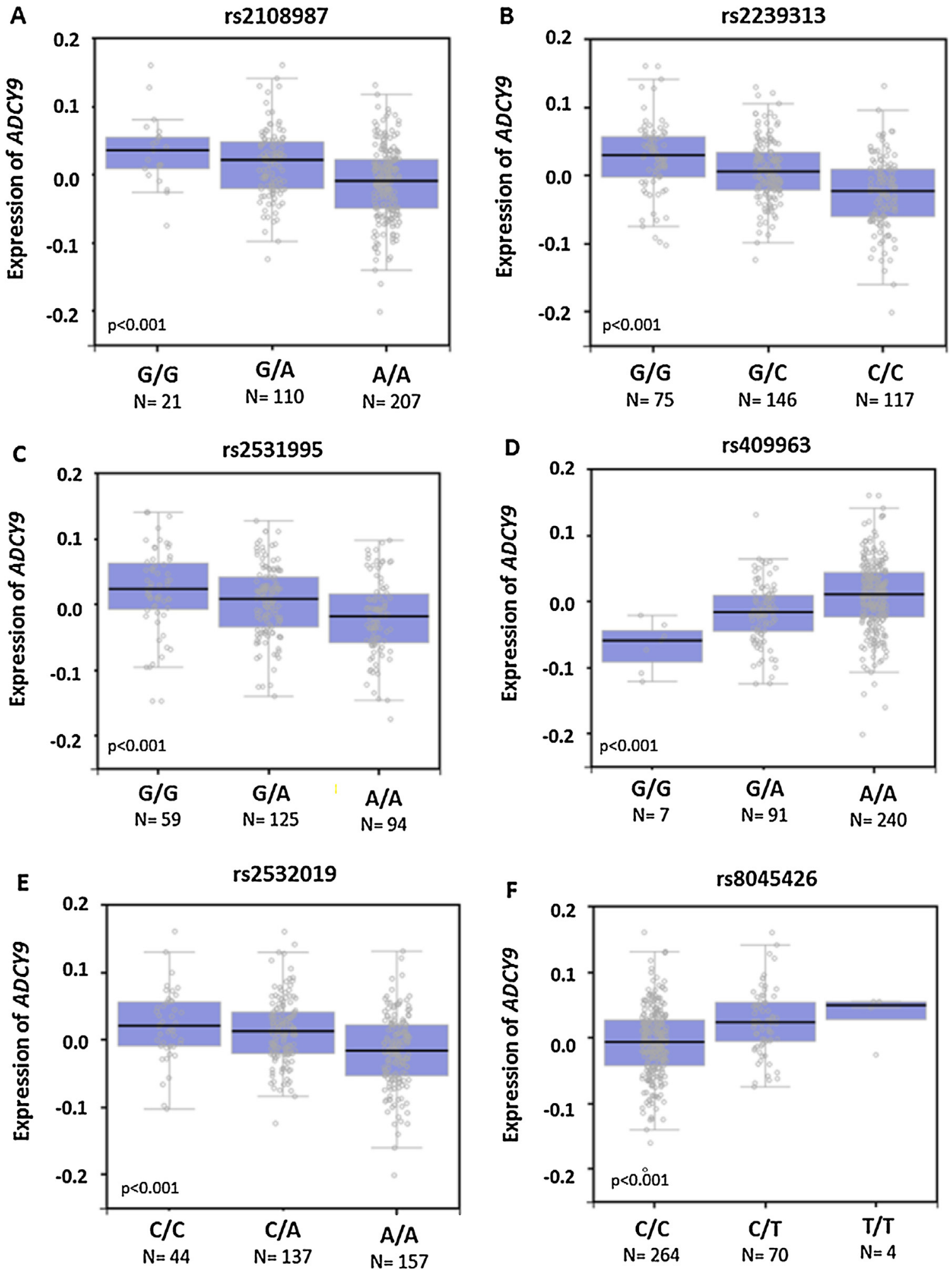

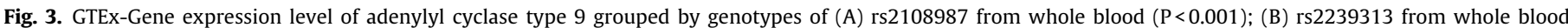

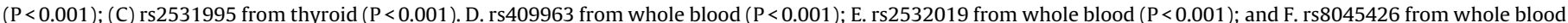
$(\mathrm{P}<0.001)$.

to allergy development (Bodor et al., 2012). Also, in mast cells, the cAMP/PKA pathway induced by E-prostanoid 2 receptors acts through suppression of IgE/antigen-dependent signals and calcium influx, reducing degranulation (Serra-pages et al., 2012) (Fig. 1).
The $G$ allele rs 2108987 was positively associated with skin test for B. germanica and unstimulated production of IL-5 and negatively associated with skin test for dog epithelium. The alternative allele $(G)$ was associated with higher $A D C Y 9$ expression than the reference allele (Fig. 3A). The SNP rs8045426 was positively associ- 
Table 6

Significant associations between $A D C Y 9$ SNPs and skin prick test by logistic regression adjusted for sex, age, helminth infections, and principal components 1 and 2.

\begin{tabular}{|c|c|c|c|c|}
\hline SNP & OR & $95 \% \mathrm{CI}$ & P value & Perm \\
\hline \multicolumn{5}{|c|}{ Skin test for at least one tested allergen } \\
\hline rs2601807 & 1.56 & $1.22-1.98$ & 0.0003 & 0.0002 \\
\hline rs8061182 & 1.42 & $1.14-1.76$ & 0.001 & 0.001 \\
\hline kgp12201986 & 1.34 & $1.11-1.62$ & 0.002 & 0.002 \\
\hline rs409963 & 1.45 & $1.12-1.87$ & 0.005 & 0.007 \\
\hline rs2531995 & 1.26 & $1.05-1.51$ & 0.013 & 0.011 \\
\hline rs2058166 & 1.33 & $1.06-1.66$ & 0.012 & 0.013 \\
\hline rs2239313 & 1.24 & $1.04-1.48$ & 0.015 & 0.013 \\
\hline rs4785951 & 1.27 & $1.06-1.52$ & 0.010 & 0.014 \\
\hline rs2444217 & 1.22 & $1.02-1.47$ & 0.034 & 0.034 \\
\hline rs2283497 & 0.80 & $0.67-0.95$ & 0.013 & 0.020 \\
\hline rs2601814 & 0.81 & $0.68-0.96$ & 0.018 & 0.018 \\
\hline rs2601831 & 0.82 & $0.69-0.98$ & 0.033 & 0.032 \\
\hline rs6500567 & 0.81 & $0.66-0.99$ & 0.043 & 0.046 \\
\hline rs73490519 & 0.64 & $0.44-0.93$ & 0.019 & 0.019 \\
\hline rs79964474 & 0.67 & $0.46-0.97$ & 0.036 & 0.041 \\
\hline \multicolumn{5}{|c|}{ Skin test for Dermatophagoides pteronyssinus } \\
\hline rs2239313 & 1.54 & $1.24-1.92$ & 0.0001 & 0.0001 \\
\hline rs2531995 & 1.43 & $1.14-1.80$ & 0.002 & 0.002 \\
\hline rs8061182 & 1.47 & $1.13-1.92$ & 0.004 & 0.003 \\
\hline rs4785951 & 1.39 & $1.11-1.75$ & 0.004 & 0.004 \\
\hline kgp12201986 & 1.38 & $1.09-1.74$ & 0.007 & 0.006 \\
\hline rs61042195 & 1.40 & $1.09-1.79$ & 0.008 & 0.007 \\
\hline rs2601807 & 1.43 & $1.06-1.92$ & 0.018 & 0.013 \\
\hline rs2058166 & 1.41 & $1.07-1.85$ & 0.015 & 0.013 \\
\hline rs2444217 & 1.28 & $1.02-1.61$ & 0.037 & 0.030 \\
\hline rs2601814 & 0.68 & $0.54-0.85$ & 0.001 & 0.0009 \\
\hline rs2532019 & 0.74 & $0.59-0.93$ & 0.009 & 0.008 \\
\hline rs73490519 & 0.48 & $0.28-0.83$ & 0.008 & 0.008 \\
\hline rs73490519 & 0.48 & $0.28-0.83$ & 0.008 & 0.008 \\
\hline rs79964474 & 0.50 & $0.29-0.86$ & 0.012 & 0.010 \\
\hline rs1045476 & 0.76 & $0.61-0.95$ & 0.014 & 0.012 \\
\hline rs9922384 & 0.66 & $0.47-0.91$ & 0.011 & 0.012 \\
\hline rs2283497 & 0.75 & $0.60-0.94$ & 0.013 & 0.014 \\
\hline rs2601831 & 0.76 & $0.61-0.96$ & 0.019 & 0.017 \\
\hline rs2601796 & 0.79 & $0.63-0.98$ & 0.035 & 0.041 \\
\hline \multicolumn{5}{|c|}{ Skin test for Blomia tropicalis } \\
\hline rs2601807 & 1.68 & $1.29-2.17$ & 0.00009 & 0.00006 \\
\hline rs2239313 & 1.29 & $1.07-1.56$ & 0.009 & 0.015 \\
\hline rs61042195 & 1.31 & $1.05-1.63$ & 0.0181 & 0.016 \\
\hline rs8061182 & 1.32 & $1.04-1.67$ & 0.022 & 0.022 \\
\hline kgp12201986 & 1.27 & $1.03-1.56$ & 0.024 & 0.020 \\
\hline rs2531995 & 1.24 & $1.01-1.51$ & 0.0373 & 0.038 \\
\hline \multicolumn{5}{|c|}{ Skin test for Periplaneta american } \\
\hline rs409963 & 1.51 & $1.09-2.08$ & 0.012 & 0.011 \\
\hline rs2058166 & 1.41 & $1.06-1.87$ & 0.017 & 0.013 \\
\hline Kgp12201986 & 1.34 & $1.05-1.70$ & 0.020 & 0.013 \\
\hline rs4785951 & 1.37 & $1.08-1.73$ & 0.008 & 0.014 \\
\hline rs2531995 & 1.32 & $1.04-1.67$ & 0.022 & 0.019 \\
\hline rs2239313 & 1.27 & $1.01-1.59$ & 0.042 & 0.039 \\
\hline rs2283497 & 0.72 & $0.57-0.92$ & 0.008 & 0.007 \\
\hline rs2532019 & 0.74 & $0.59-0.94$ & 0.014 & 0.018 \\
\hline rs2601814 & 0.77 & $0.61-0.97$ & 0.027 & 0.032 \\
\hline rs9922384 & 0.70 & $0.50-0.98$ & 0.040 & 0.043 \\
\hline rs6500567 & 0.75 & $0.57-0.99$ & 0.047 & 0.048 \\
\hline \multicolumn{5}{|c|}{ Skin test for Blatella germanica } \\
\hline rs2108987 & 1.54 & $1.10-2.14$ & 0.011 & 0.015 \\
\hline rs2601798 & 1.64 & $1.06-2.53$ & 0.027 & 0.022 \\
\hline rs13337675 & 0.58 & $0.37-0.89$ & 0.015 & 0.018 \\
\hline \multicolumn{5}{|c|}{ Skin test for dog epithelium } \\
\hline rs2239313 & 2.91 & $1.33-6.37$ & 0.007 & 0.004 \\
\hline rs4785951 & 2.24 & $1.05-4.81$ & 0.038 & 0.022 \\
\hline rs2108987 & 0.12 & $0.02-0.93$ & 0.042 & 0.021 \\
\hline \multicolumn{5}{|c|}{ Skin test for cat epithelium } \\
\hline rs7196832 & 3.02 & $1.10-8.29$ & 0.032 & 0.029 \\
\hline
\end{tabular}

Table 7

Association between ADCY9 SNPs and unstimulated production of IL- 5 and IL-13 by logistic regression adjusted for sex, age, helminth infections, and principal components 1 and 2 .

\begin{tabular}{lllll}
\hline SNP & OR & 95\% CI & P value & Perm \\
\hline \multicolumn{2}{l}{ Unstimulated production of IL-5 } & & & \\
$\quad$ rs8045426 & 1.86 & $1.09-3.15$ & 0.022 & 0.011 \\
rs2601798 & 1.92 & $1.14-3.25$ & 0.015 & 0.012 \\
rs2108987 & 1.73 & $1.14-2.60$ & 0.009 & 0.014 \\
rs7196832 & 1.56 & $1.06-2.27$ & 0.022 & 0.020 \\
rs13337675 & 0.46 & $0.25-0.86$ & 0.015 & 0.012 \\
Unstimulated production of IL-13 & & & \\
rs2531995 & 1.24 & $1.03-1.49$ & 0.021 & 0.026 \\
rs8045426 & 1.41 & $1.04-1.89$ & 0.025 & 0.026 \\
rs1045476 & 0.83 & $0.70-0.99$ & 0.034 & 0.037 \\
\hline
\end{tabular}

ated with unstimulated production of IL-5 and IL-13 and negatively associated with skin test for $B$. tropicalis. The alternative allele (T) had high expression levels (Fig. 3F).

Although these 2 SNPs have a divergent association, this was explained by Dehzad et al. (2016), where Th2 cell effector functions remained unaffected due the ability of Th2 cells to produce cytokines, which sustain their own proliferation, even in the absence of responsiveness to IL-2. In addition, a high cAMP content failed to efficiently block Th2-mediated IL-4 production, whereas IL-2 secretion and proliferation of Th1 cells were inhibited (Dehzad et al., 2016).

The rs2601798 and rs7196832 SNPs were positively associated with skin test for B. germanica and cat epithelium, respectively, and both were positively associated with unstimulated production of IL-5. The A allele of rs1045476 was negatively associated with skin test for $D$. pteronyssinus and unstimulated production of IL13 , and the $G$ allele of rs13337675 was negatively associated with skin test for B. germanica and unstimulated production of IL-5. Th2 cells orchestrate asthmatic inflammation through the secretion of a series of cytokines, particularly IL-4, IL-9, IL-5, and IL-13; these cytokines seem to be a main factor determining the degree of airway inflammation and hyperresponsiveness (Akbari et al., 2003). IL-4 and IL-13 are involved in the isotype switch from IgM to IgE, the antibody responsible for classic allergy and implicated in the pathophysiology of allergic asthma (Akbari et al., 2003). Also, IL-13 increases mucus production and induces airway hyperreactivity, a cardinal feature of asthma (Akbari et al., 2003). IL-5 plays a major role in the regulation of eosinophil formation, growth, differentiation, recruitment, and survival (Deo et al., 2010; Kouro and Takatsu, 2009).

The rs9922384 was negatively associated with skin test for $D$. pteronyssinus and $P$. americana. The SNP was localized in the promoter region of the $A D C Y 9$ gene with an intron variant function. This polymorphism shows a $2 \mathrm{~b}$ score in the RegulomeDB database, which was related to binding transcription factors to DNA and, potentially, correlated to the level of ADCY9 expressed. Other negatively associated polymorphisms were the SNP rs2283497 with atopic asthma, skin test for at least one tested allergen $(\geq 3 \mathrm{~mm})$, specific skin test for $D$. pteronyssinus and IgE for $D$. pteronyssinus, $P$. americana, and B. tropicalis; the SNP rs4785953 with the skin test for at least one tested allergen ( $\geq 3 \mathrm{~mm}$ ) and $\mathrm{IgE}$ for $D$. pteronyssinus and $B$. tropicalis; the SNP rs2601777 with at least one IgE, specifically, for $B$. tropicalis; the rs 2601831 with the skin test for at least one allergen and to $D$. pteronyssinus; rs432166 with IgE for $B$. tropicalis and IgE for at least one allergen; rs6500567 with IgE for $D$. pteronyssinus, the skin test for $P$. americana, and for at least one allergen; and rs73490519 and rs79964474 with the skin test for $D$. pteronyssinus and for at least one allergen. These polymorphisms likely protect the subject from asthma or the atopic phenotype; data about these SNPs are lacking. 
The SNP rs2444217 is positively associated with the skin test for $D$. pteronyssinus and at least one tested allergen $(\geq 3 \mathrm{~mm})$ and IgE for B. tropicalis. This SNP presents a DB score of 2a that possesses sequence motifs. These are a short sequences, recurring patterns in DNA that have a biological function. They indicate sequence-specific binding sites for proteins, such as nucleases and transcription factors (TF). Sequence motifs are involved in important processes at the RNA level, including ribosome binding, mRNA processing (splicing, editing, polyadenylation) (Boyle et al., 2012) (Patrik, 2006). The SNP rs2444217 is directly correlated with $A D C Y$ expression. Other polymorphisms are associated positively, such as the kgp12201986 with atopic asthma, the skin test for $D$. pteronyssinus, P. americana, and B. tropicalis, IgE for B. tropicalis and IgE, and the skin test for at least one allergen. The SNP rs2058166 is associated with the skin test for $D$. pteronyssinus and $P$. americana and for at least one allergen; rs2601807 with the skin test for D. pteronyssinus, B. tropicalis, and for at least one allergen; rs4785947 with atopic asthma and sIgE to B. tropicalis; rs4785951 with the skin test for $D$. pteronyssinus, $P$. americana, dog epithelium, and for at least one allergen; rs61042195 with the skin test for $D$. pteronyssinus and $B$. tropicalis; and finally, rs8061182 is positively associated with atopic asthma and the skin test for at least one tested allergen ( $\geq 3 \mathrm{~mm}$ ), specifically, for $D$. pteronyssinus and $B$. tropicalis. These polymorphisms may increase the risk of atopic asthma; data in the literature are scarce for these SNPs.

\section{Conclusions}

To conclude, polymorphisms in the ADCY9 gene may play a role in asthma and/or allergy. Although a relationship has not been demonstrated for all of these SNPs with adenylyl cyclase expression, we believe that some $A D C Y 9$ polymorphisms in atopic subjects decrease adenylyl cyclase expression, leading to less cAMP production and consequently, deficient immunomodulatory activity. Moreover, two SNPs showed negative associations with allergic markers and, on the other hand, a positive association with asthma possibly a non-atopic asthma. Therefore, it is necessary to replicate this study in other populations as well as to perform experiments that elucidate the mechanism whereby this genotype interferes in the immune response leading to asthma and allergies.

\section{Appendix A. Supplementary data}

Supplementary data associated with this article can be found, in the online version, at http://dx.doi.org/10.1016/j.molimm.2017. 01.001 .

\section{References}

Akbari, O., Stock, P., Dekruyff, R.H., Umetsu, D.T., 2003. Role of regulatory T cells in allergy and asthma. Eur. PMC 15, 627-633, http://dx.doi.org/10.1016/j.coi. 2003.09.012.

Alcantara-Neves, N.M., Veiga, V., Cavalcante, C., 2011. The effect of single and multiple infections on atopy and wheezing in children. J. Allergy Clin. Immunol. 129, 359-367, http://dx.doi.org/10.1016/j.jaci.2011.09.015.

Barreto, M.L., Cunha, S.S., Alcântara-neves, N., Carvalho, L.P., Cruz, Á.A., Stein, R.T., Genser, B., Cooper, P.J., Rodrigues, L.C., 2006. Risk factors and immunological pathways for asthma and other allergic diseases in children: background and methodology of a longitudinal study in a large urban center in Northeastern Brazil (Salvador-SCAALA study). BMC Pulm. Med., 10, http://dx.doi.org/10. 1186/1471-2466-6-15.
Barrett, J.C., Fry, B., Maller, J., Daly, M.J., 2005. Haploview: analysis and visualization of LD and haplotype maps. Bioinformatics 21, 263-265, http://dx. doi.org/10.1093/bioinformatics/bth457.

Bodor, J., Bopp, T., Vaeth, M., Klein, M., Serfling, E., Hünig, T., Becker, C., Schild, H. Schmitt, E., 2012. Cyclic AMP underpins suppression by regulatory T cells. Eur. J. Immunol. 42, 1375-1384, http://dx.doi.org/10.1002/eji.201141578.

Boyle, A.P., Hong, E.L., Hariharan, M., Cheng, Y., Schaub, M.A., Kasowski, M. Karczewski, K.J., Park, J., Hitz, B.C., Weng, S., Cherry, J.M., Snyder, M., 2012. Annotation of functional variation in personal genomes using RegulomeDB. Genome Res., 1790-1797, http://dx.doi.org/10.1101/gr.137323.112.

Costa, G.N.O., Dudbridge, F., Fiaccone, R.L., Silva, T.M., Conceição, J.S., Strina, A., Figueiredo, C.A., Magalhães, W.C.S., Rodrigues, M.R., Gouveia, M.H., Kehdy, F.S.G., Horimoto, A.R.V.R., Horta, B., Burchard, E.G., Pino-yanes, M., Del, B., Navarro, R., Romieu, I., Hancock, D.B., London, S., Lima-costa, M.F., Pereira, A.C. Tarazona, E., Rodrigues, L.C., Barreto, M.L., 2015. A genome-wide association study of asthma symptoms in Latin American children. BMC Genet., 1-11, http://dx.doi.org/10.1186/s12863-015-0296-7.

Dehzad, N., Bopp, T., Reuter, S., Martin, H., Ulges, A., Stassen, M., Schild, H., Buhl, R. Schmitt, E., Buhl, R., Schmitt, E., 2016. Regulatory T cells more effectively suppress Th1-induced airway inflammation compared with th2. J. Immunol. 186, 2238-2244, http://dx.doi.org/10.4049/jimmunol.1002027.

Deo, S.S., Mistry, K.J., Kakade, A.M., Niphadkar, P., 2010. T type cytokines in IgE mediated allergy and asthma. Lung India 27, h2, http://dx.doi.org/10.4103/ 0970-2113.63609.

Global Asthma Network, 2014. The Global Asthma Report 2014. ISBN: 978-0-473-29125-9978-0-473-29126-6 (ELECTRONIC)

Holgate, S.T., 2012. Innate and adaptive immune responses in asthma. Nat. Med. 18, 673-684, http://dx.doi.org/10.1038/nm.2731, review.

Huang, B., Zhao, J., Lei, Z., Shen, S., Li, D., Shen, G.-X., Zhang, G.-M., Feng, Z.-H., 2009 miR-142-3p restricts cAMP production in CD4 + CD25-T cells and CD4 + CD25+ TREG cells by targeting AC9 mRNA. EMBO Rep. 10, 180-185, http://dx.doi.org/ 10.1038/embor.2008.224.

Kouro, T., Takatsu, K., 2009. IL-5- and eosinophil-mediated inflammation: from discovery to therapy, Int. Immunol., 21, 1303-1309. 10.1093/intimm/dxp102.

Lage-Castellanos, A., Martínez-Montes, E., Hernández-Cabrera, J., Galán, L., 2010. False discovery rate and permutation test: an evaluation in ERP data analysis.Stat Med., 29(1):63-74 10.1002/sim.3784.

Miller, S.M., Ortega, V.E., 2013. Pharmacogenetics and the development of personalized approaches for combination therapy in asthma. Curr. Allergy Asthma Rep. 13, 443-452, http://dx.doi.org/10.1007/s11882-013-0372-x.

Mosenden, R., Taskén, K., 2011. Cyclic AMP-mediated immune regulation-overview of mechanisms of action in T cells. Cell Signal. 23 1009-1016, http://dx.doi.org/10.1016/j.cellsig.2010.11.018.

Ortega, V.E., 2015. Asthma pharmacogenetics and the development of genetic profiles for personalized medicine, Pharmgenomics Pers Med.; 8:9-22. 10.2147/PGPM.S52846.

Patrik, D., 2006. What are DNA sequence motifs? Nat. Biotechnol. 24, 423-425 $10.1038 /$ nbt0406-42324, 423-425.

Purcell, S., Neale, B., Todd-brown, K., Thomas, L., Ferreira, M.A.R., Bender, D., Maller J., Sklar, P., Bakker, P.I.W. De, Daly, M.J., Sham, P.C., 2007. REPORT PLINK: A Tool Set for Whole-Genome Association and Population-Based Linkage Analyses, Am J Hum Genet. 81, 559-575. 10.1086/519795.

Serra-pages, M., Olivera, A., Torres, R., Picado, C., Mora F., De, 2012. E-prostanoid 2 receptors dampen mast cell degranulation via cAMP/PKA-mediated suppression of IgE-dependent signaling, J Leukoc Biol. 92, 1155-1165. 10.1189/jlb.0212109.

Sunahara, R.K., Taussig, R., Arbor, A., 2002. Isoforms of Mammalian Adenylyl Cyclase: multiplicities of signaling. Mol. Interv. 168-184. 10.1124/mi.2.3.168.

Tantisira, K.G., Small, K.M., Litonjua, A. a., Weiss, S.T., Liggett, S.B., 2005. Molecular properties and pharmacogenetics of a polymorphism of adenylyl cyclase type 9 in asthma: interaction between beta-agonist and corticosteriod pathways. Hum. Mol. Genet. 14, 1671-1677, http://dx.doi.org/10.1093/hmg/ddi175.

The GTEx Consortium, 2014. The Genotype-Tissue Expression (GTEx) project 45 , 580-585. 10.1038/ng.2653. The.

Verjans, E., Ohl, K., Reiss, L.K., Wijk, F., Van, Antonaneta, A., Wiener, A., Yu, Y., Rieg, A.D., Gaertner, V.D., Roth, J., Knol, E., Kabesch, M., Wagner, N., Martin, C., Tenbrock, K., 2015. The cAMP response element modulator (CREM) regulates $\mathrm{T}$ H 2 mediated inflammation, Oncotarget. 6, 38538-38551, 10.18632 /oncotarget.6041.

Wang, Y., Zhao, L.P., Dudoit, S., 2006. A fine-scale linkage-disequilibrium measure based on length of haplotype sharing. Am. J. Hum. Genet. 7878, 615-628, http://dx.doi.org/10.1086/502632.

Yoshida, N., Comte, D., Mizui, M., Otomo, K., Rosetti, F., Mayadas, T.N., Crispı×n, J.C., Bradley, S.J., Koga, T., Kono, M., Karampetsou, M.P., Kyttaris, V.C., Tsokos C. George, Tenbrock, K., 2016. ICER is requisite for Th17 differentiation, Nat Commun, 7:12993.10.1038/ncomms12993. 